\title{
Dynamical barriers for the random ferromagnetic Ising model on the Cayley tree : traveling-wave solution of the real space renormalization flow
}

\author{
Cécile Monthus and Thomas Garel \\ Institut de Physique Théorique, CNRS and CEA Saclay, 91191 Gif-sur-Yvette, France
}

\begin{abstract}
We consider the stochastic dynamics near zero-temperature of the random ferromagnetic Ising model on a Cayley tree of branching ratio $K$. We apply the Boundary Real Space Renormalization procedure introduced in our previous work (C. Monthus and T. Garel, J. Stat. Mech. P02037 (2013)) in order to derive the renormalization rule for dynamical barriers. We obtain that the probability distribution $P_{n}(B)$ of dynamical barrier for a subtree of $n$ generations converges for large $n$ towards some traveling-wave $P_{n}(B) \simeq P^{*}(B-n v)$, i.e. the width of the probability distribution remains finite around an average-value that grows linearly with the number $n$ of generations. We present numerical results for the branching ratios $K=2$ and $K=3$. We also compute the weak-disorder expansion of the velocity $v$ for $K=2$.
\end{abstract}

\section{INTRODUCTION}

The stochastic dynamics of classical disordered spin systems turns out to be extremely slow in the whole lowtemperature phase (see for instance the books [1, 2] and references therein). The reason is that the system tends to remain trapped in valleys of configurations on various scales. Within the point of view of dynamical simulations, this problem has been called the 'futility' problem [3] : the number of distinct configurations visited during the simulation remains very small with respect to the accepted moves. The reason is that the system visits over and over again the same configurations within a given valley before it is able to escape towards another valley.

As a consequence, a natural idea is to formulate some appropriate renormalization procedure for dynamical barriers. For random walks in random media, Strong Disorder Renormalization rules have been formulated in real space [46]. The direct generalization of this approach to many-body systems leads to Strong Disorder Renormalization formulated in configuration space [7], because the renormalization concerns the master equation of the dynamics defined in configuration space. These Strong Disorder RG approaches are perfect to describe correctly the hierarcal organization of valleys within valleys. However, from a numerical point of view, since the size of the configuration space grows exponentially with the number of degrees of freedom of the many-body system, this approach can be applied numerically only for small sizes [7].

In a recent work [8], we have thus introduced a different renormalization approach formulated in real space : using the standard mapping between the detailed-balance dynamics of classical Ising models and some quantum Hamiltonian, we have derived appropriate real-space renormalization rule for this quantum Hamiltonian. We have solved explicitly the renormalization flow for the random ferromagnetic chain [8] , for the pure Ising model on the Cayley tree [8], and for the hierarchical Dyson Ising model [9]. In the present paper, we study the case of the random ferromagnetic Ising model on the Cayley tree.

The paper is organized as follows. In section [I] we explain how the Real Space Renormalization approach of [8] can be applied to the stochastic dynamics of the random ferromagnetic Ising model on the Cayley tree. In section III. we derive the renormalization rules for dynamical barriers near zero temperature. In section IV] we study the renormalization flow for the probability distribution of dynamical barriers. The cases of branching ratio $K=2$ and $K=3$ are discussed respectively in sections $\mathrm{V}$ and $\mathrm{VI}$ with numerical results on the traveling-wave statistics of dynamical barriers. Section VII summarizes our conclusions.

\section{REAL SPACE RENORMALIZATION APPROACH}

\section{A. Model and notations}

We consider the random ferromagnetic Ising model with the classical energy

$$
U(\mathcal{C})=-\sum_{i<j} J_{i j} S_{i} S_{j}
$$

defined on a Cayley tree of branching ratio $K$ with $N$ generations, and with free boundary conditions on all the boundary spins. The coordinence of non-boundary spins is thus $(K+1)$. The couplings $J_{i j}$ are independent random positive variables drawn with some law $\rho(J)$. 
The stochastic dynamics is defined by the master equation

$$
\frac{d P_{t}(\mathcal{C})}{d t}=\sum_{\mathcal{C}^{\prime}} P_{t}\left(\mathcal{C}^{\prime}\right) W\left(\mathcal{C}^{\prime} \rightarrow \mathcal{C}\right)-P_{t}(\mathcal{C}) W_{\text {out }}(\mathcal{C})
$$

that describes the time evolution of the probability $P_{t}(\mathcal{C})$ to be in configuration $\mathcal{C}$ at time t. The notation $W\left(\mathcal{C}^{\prime} \rightarrow \mathcal{C}\right)$ represents the transition rate per unit time from configuration $\mathcal{C}^{\prime}$ to $\mathcal{C}$, and

$$
W_{\text {out }}(\mathcal{C}) \equiv \sum_{\mathcal{C}^{\prime}} W\left(\mathcal{C} \rightarrow \mathcal{C}^{\prime}\right)
$$

represents the total exit rate out of configuration $\mathcal{C}$. We will focus here on single spin-flip dynamics satisfying detailed balance

$$
e^{-\beta U(\mathcal{C})} W\left(\mathcal{C} \rightarrow \mathcal{C}^{\prime}\right)=e^{-\beta U\left(\mathcal{C}^{\prime}\right)} W\left(\mathcal{C}^{\prime} \rightarrow \mathcal{C}\right)
$$

where the transition rate corresponding to the flip of a single spin $S_{k}$ reads

$$
W\left(S_{k} \rightarrow-S_{k}\right)=G^{i n i}\left[h_{k}=\sum_{i \neq k} J_{i k} S_{i}\right] e^{-\beta S_{k}\left[\sum_{i \neq k} J_{i k} S_{i}\right]}
$$

$G^{i n i}\left[h_{k}\right]$ is an arbitrary positive even function of the local field $h_{k}=\sum_{i \neq k} J_{i k} S_{i}$. For instance, the Glauber dynamics corresponds to the choice

$$
G_{\text {Glauber }}^{\text {ini }}[h]=\frac{1}{2 \cosh (\beta h)}
$$

\section{B. Associated quantum Hamiltonian}

As is well-known, the master equation of Eq. 2 with the choice of Eq. 5] can be mapped via the similarity transformation $P_{t}(\mathcal{C}) \equiv e^{-\frac{\beta}{2} U(\mathcal{C})} \psi_{t}(\mathcal{C})$ onto a Schrödinger equation with the following quantum Hamiltonian involving Pauli matrices $\left(\sigma^{x}, \sigma^{z}\right)[8,10-16]$

$$
\mathcal{H}=\sum_{k} G^{i n i}\left[\sum_{i \neq k} J_{i k} \sigma_{i}^{z}\right]\left(e^{-\beta \sigma_{k}^{z}\left(\sum_{i \neq k} J_{i k} \sigma_{i}^{z}\right)}-\sigma_{k}^{x}\right)
$$

Note that in the high-temperature limit $\beta \rightarrow 0$, the quantum Hamiltonian of Eq. 7 for the Glauber dynamics of Eq. 6] reduces to the standard transverse-field Ising model

$$
\mathcal{H} \underset{\beta \rightarrow 0}{\simeq} N+\sum_{k=1}^{\sim}\left(-\beta \sigma_{k}^{z}\left(\sum_{i \neq k} J_{i k} \sigma_{i}^{z}\right)-\sigma_{k}^{x}\right)
$$

When the coupling $J_{i j}$ are random, the low-energy physics is then well described by the Strong Disorder RG procedure valid both in one dimension [17] and in higher dimensions $d>1$ [18 20] (see [21] for a review). However here we are interested into the opposite limit of very low temperature where $\beta \rightarrow+\infty$, where one cannot linearize the exponentials in the quantum Hamiltonians of Eq. 7]. We have explained in [8] how to define appropriate real-space renormalization rules for this type of quantum Hamiltonian in the opposite limit of very low temperature. In the following, we recall the main idea for the case of the Cayley tree geometry.

\section{Reminder on the Boundary Real Space Renormalization on a Cayley tree}

For a model defined on a Cayley tree, it is natural to define a boundary renormalization procedure in order to keep the tree topology unchanged, and to obtain recurrence equations. For instance for the Random Transverse Field Ising Model of Eq. 8, this idea has been used either within the Quantum Cavity Approach [22 24] or within the Boundary RG approach [25]. 


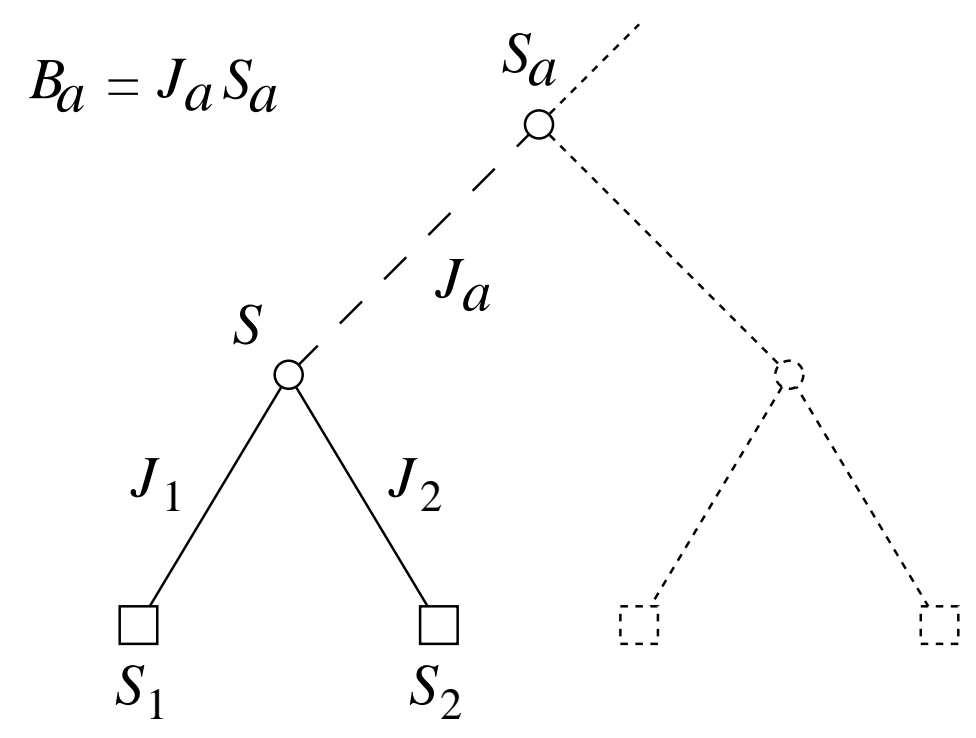

FIG. 1: Basic step of the Boundary Real Space Renormalization for a Cayley tree of branching ratio $K=2:$ the quantum Hamiltonian of Eq. 9 involves $K+1=3$ spins, namely the two renormalized boundary spins $\left(S_{1}, S_{2}\right)($ that represent sub-trees) whose renormalized dynamics is described by renormalized amplitudes $\left(G_{1}, G_{2}\right)$, and their common ancestor $S$, whose dynamics is still described by the initial amplitude $G^{i n i}[h]$. The further ancestor $S_{a}$ is not taken into account as a quantum spin, but only through the external field $B_{a}=J_{a} S_{a}$ seen by the spin $S$.

For the Hamiltonian of Eq. 7, a boundary spin $S_{i}$ is connected to a single ancestor spin $S$ via some random positive coupling $J_{i}>0$, so the absolute value of its local field $h_{i}=J_{i} S$ takes the single value $J_{i}$. As a consequence, the function $G^{i n i}\left(h_{i}\right)$ reduces to the number $G^{i n i}\left(J_{i}\right)$. As explained in [8] for the case of the pure Ising model, it is thus convenient to define a Boundary Real Space Renormalization as follows. The basic renormalization step concerns $K$ renormalized boundary spins $\left(S_{1}, S_{2}, . ., S_{K}\right)$ whose renormalized dynamics is described by some renormalized amplitudes $G_{i}$ (which are numbers and not operators) and their common ancestor spin $S$ whose dynamics is still described by the initial amplitude $G^{i n i}[h]$ involving also the external field $B_{a}=J_{a} S_{a}$ induced by its next ancestor spin $S_{a}$. So we have to study the following effective Hamiltonian for these $(K+1)$ spins $\left(S_{1}, . ., S_{K}, S\right)$

$$
H_{K+1}=G^{i n i}\left[\sum_{i=1}^{K} J_{i} \sigma_{i}^{z}+B_{a}\right]\left(e^{-\beta \sigma^{z}\left(\sum_{i=1}^{K} J_{i} \sigma_{i}^{z}+B_{a}\right)}-\sigma^{x}\right)+\sum_{i=1}^{K} G_{i}\left(e^{\left.-\beta \sigma_{i}^{z} J_{i} \sigma^{z}\right)}-\sigma_{i}^{x}\right)
$$

The physical meaning of the amplitude $G_{i}$ of the renormalized boundary spin $S_{i}$ that represents a whole sub-tree, is that the largest relaxation time for this isolated sub-tree reads (see detailed explanations in [8])

$$
t_{i}^{\text {relax }}=\frac{1}{2 G_{i}}
$$

Near zero temperature, the largest relaxation time is simply the equilibrium time $t_{i}^{e q}$ needed to flip between the two renormalized states states $S_{i}=+1$ and $S_{i}=-1$ representing the two ferromagnetic ground states of the corresponding sub-tree, so the corresponding dynamical barrier $\mathcal{B}_{i}$ is defined by the exponential behavior

$$
t_{i}^{\text {relax }}=\frac{1}{2 G_{i}} \underset{\beta \rightarrow+\infty}{\propto} e^{\beta \mathcal{B}_{i}}
$$

After the first renormalization step, the renormalized dynamical barrier $\mathcal{B}_{i}$ are expected to grow, so that the renormalized amplitudes $G_{i}$ will be extremely small. The most appropriate approach is then a perturbative analysis in the parameters $G_{i}$ that may be summarized as follows (see [8] for more details) :

(i) When $G_{i}=0$ for $i=1,2, . ., K$, the spins $\left(S_{1}, . ., S_{K}\right)$ cannot flip and are thus frozen. So the $2^{K}$ states

$$
\left|v_{0}^{S_{1}, S_{2}, . ., S_{K}}>\equiv \prod_{j=1}^{K}\right| S_{j}>\sum_{S= \pm} \frac{e^{\frac{\beta}{2} S\left(\sum_{i=1}^{K} J_{i} S_{i}+B_{a}\right)}}{\sqrt{2 \cosh \beta\left(\sum_{i=1}^{K} J_{i} S_{i}+B_{a}\right)}} \mid S>
$$


are zero-energy states of Hamiltonian of Eq. 9 when $G_{i}=0$ for any function $G^{i n i}[h]$ since one has

$$
\left(e^{-\beta \sigma^{z}\left(\sum_{i=1}^{K} J_{i} \sigma_{i}^{z}+B_{a}\right)}-\sigma^{x}\right) \mid v_{0}^{S_{1}, S_{2}, . ., S_{K}}>=0
$$

The physical interpretation is that the spin $S$ is at equilibrium with respect to the frozen spins $\left(S_{1}, \ldots, S_{K}\right)$. The other $2^{K}$ states have a finite energy for $G_{i}=0$.

(ii) When the amplitudes $G_{i}$ for $i=1,2, . ., K$ are small, we need to diagonalize the perturbation within the subspace spanned by the $2^{K}$ vectors $\mid v_{0} S_{1}, S_{2}, \ldots S_{K}>$ of Eq. 12. We look for an eigenstate via the linear combination

$$
\left|u_{\lambda}>=\sum_{S_{1}= \pm, S_{2}= \pm, \ldots, S_{K}= \pm} T_{\lambda}\left(S_{1}, S_{2}, \ldots, S_{K}\right)\right| v_{0}^{S_{1}, S_{2}, \ldots S_{K}}>
$$

The eigenvalue equation $0=\left(H_{K+1}-\lambda\right) \mid u_{\lambda}>$ reads

$$
\begin{aligned}
0 & =\left[\sum_{i=1}^{K} G_{i} \frac{2 \cosh \beta\left(\sum_{j \neq i} J_{j} S_{j}+B_{a}\right)}{2 \cosh \beta\left(\sum_{j=1}^{K} J_{j} S_{j}+B_{a}\right)}-\lambda_{1}\right] T_{\lambda_{1}}\left(S_{1},, \ldots, S_{K}\right) \\
& -\sum_{i=1}^{K} G_{i} \frac{2 \cosh \beta\left(\sum_{j \neq i} J_{j} S_{j}+B_{a}\right)}{\sqrt{2 \cosh \beta\left(\sum_{j \neq i} J_{j} S_{j}-J_{i} S_{i}+B_{a}\right)} \sqrt{2 \cosh \beta\left(\sum_{j \neq i} J_{j} S_{j}+J_{i} S_{i}+B_{a}\right)}} T_{\lambda_{1}}\left(S_{1}, . .,-S_{i}, \ldots, S_{K}\right)
\end{aligned}
$$

(iii) As explained in detail in [8], one obtains that near zero-temperature, the Hamiltonian of Eq. 9 can be renormalized onto the single spin effective Hamiltonian

$$
H \simeq G_{R}\left(e^{-\beta B_{a} \sigma_{R}^{z}}-\sigma_{R}^{x}\right)
$$

for the renormalized spin (describing the full ferromagnetic states)

$$
\begin{aligned}
\left|S_{R}=+>\equiv\left(\prod_{j=1}^{K} \mid S_{j}=+>\right)\right| S=+> \\
\left|S_{R}=->\equiv\left(\prod_{j=1}^{K} \mid S_{j}=->\right)\right| S=->
\end{aligned}
$$

with the renormalized amplitude (using Eq 34)

$$
G_{R}=\frac{\lambda_{1}}{2 \cosh \beta J_{a}}
$$

where $\lambda_{1}$ is the first non-vanishing eigenvalue of the system of Eq. 15]

In the pure case studied in [8], i.e. when all couplings $J_{i}$ take the same value $J$, and where all amplitudes $G_{i}$ take the same value $G$, we have solved Eq 15 by taking into account the symmetry between the $K$ branches. Here in the disordered case, the $K$ branches are not equivalent anymore, but we can nevertheless derive an explicit renormalization rule for $G_{R}$, as explained in the following section.

\section{RENORMALIZATION RULE FOR DYNAMICAL BARRIERS}

\section{A. Eigensystem for $\lambda_{1}$}

To see more clearly the meaning of the eigensystem of Eq. 15, it is convenient to introduce

$$
T_{0}\left(S_{1}, . ., S_{K}\right) \equiv \sqrt{2 \cosh \beta\left(\sum_{i=1}^{K} J_{i} S_{i}+B_{a}\right)}
$$

which solve the system of Eq. 15 for $\lambda=0$, and the ratios

$$
A\left(S_{1}, . ., S_{K}\right) \equiv \frac{T_{\lambda_{1}}\left(S_{1}, . ., S_{K}\right)}{T_{0}\left(S_{1}, . ., S_{K}\right)}
$$


so that Eq 15 reads

$$
0=\left[\sum_{i=1}^{K} G_{i} \frac{T_{0}^{2}\left(S_{1}, ., S_{i}=0, ., S_{K}\right)}{T_{0}^{2}\left(S_{1}, ., S_{i}, ., S_{K}\right)}-\lambda\right] A\left(S_{1}, . . ., S_{K}\right)-\sum_{i=1}^{K} G_{i} \frac{T_{0}^{2}\left(S_{1}, ., S_{i}=0, ., S_{K}\right)}{T_{0}^{2}\left(S_{1}, ., S_{i}, ., S_{K}\right)} A\left(S_{1}, . .,-S_{i}, . ., S_{K}\right)
$$

To compute the lowest non-vanishing eigenvalue $\lambda_{1}$ of Eq. 15. it is consistent to set $\lambda_{1}=0$ in all equations except at the two extreme cases where all spins have the same value, either $S_{a}$ or $-S_{a}$, where $B_{a} \equiv J_{a} S_{a}$. The two extreme components should be orthogonal to Eq19 and thus read

$$
\begin{array}{r}
T_{\lambda_{1}}\left(S_{a}, S_{a}, \ldots, S_{a}\right)=T_{0}\left(-S_{a},-S_{a}, \ldots,-S_{a}\right)=\sqrt{2 \cosh \beta\left(\sum_{i=1}^{K} J_{i}-J_{a}\right)} \simeq e^{\frac{\beta}{2}\left(\sum_{i=1}^{K} J_{i}-J_{a}\right)} \\
T_{\lambda_{1}}\left(-S_{a},-S_{a}, \ldots,-S_{a}\right)=-T_{0}\left(S_{a}, S_{a}, \ldots, S_{a}\right)=-\sqrt{2 \cosh \beta\left(\sum_{i=1}^{K} J_{i}+J_{a}\right)} \simeq-e^{\frac{\beta}{2}\left(\sum_{i=1}^{K} J_{i}+J_{a}\right)}
\end{array}
$$

i.e. the two extreme values of the ratios of Eq. 20

$$
\begin{aligned}
A\left(S_{a}, S_{a}, \ldots, S_{a}\right) & =\frac{T_{0}\left(-S_{a},-S_{a}, \ldots,-S_{a}\right)}{T_{0}\left(S_{a}, S_{a}, \ldots, S_{a}\right)}=\frac{\sqrt{2 \cosh \beta\left(\sum_{i=1}^{K} J_{i}-J_{a}\right)}}{\sqrt{2 \cosh \beta\left(\sum_{i=1}^{K} J_{i}+J_{a}\right)}} \simeq e^{-\beta J_{a}} \\
A\left(-S_{a},-S_{a}, \ldots,-S_{a}\right) & =-\frac{T_{0}\left(S_{a}, S_{a}, \ldots, S_{a}\right)}{T_{0}\left(-S_{a},-S_{a}, \ldots,-S_{a}\right)}=-\frac{\sqrt{2 \cosh \beta\left(\sum_{i=1}^{K} J_{i}+J_{a}\right)}}{\sqrt{2 \cosh \beta\left(\sum_{i=1}^{K} J_{i}-J_{a}\right)}} \simeq e^{\beta J_{a}}
\end{aligned}
$$

satisfy Eq21 with $\lambda_{1}$. So $\lambda_{1}$ can be computed near zero temperature as

$$
\begin{aligned}
\lambda_{1} & =\sum_{i=1}^{K} G_{i} \frac{T_{0}^{2}\left(S_{a}, S_{a}, \ldots, S_{a}, S_{i}=0, S_{a}, S_{a}, \ldots, S_{a}\right)}{T_{0}^{2}\left(S_{a}, S_{a}, \ldots, S_{a}\right)}\left(1-\frac{A\left(S_{a}, S_{a}, \ldots, S_{a}, S_{i}=-S_{a}, S_{a}, S_{a}, \ldots, S_{a}\right)}{A\left(S_{a}, S_{a}, \ldots, S_{a}\right)}\right) \\
& \simeq \sum_{i=1}^{K} G_{i} e^{-\beta J_{i}}\left(1-\frac{A\left(S_{a}, S_{a}, \ldots, S_{a}, S_{i}=-S_{a}, S_{a}, S_{a}, \ldots, S_{a}\right)}{A\left(S_{a}, S_{a}, \ldots, S_{a}\right)}\right)
\end{aligned}
$$

whereas all non-extreme values satisfy Eq 21 with $\lambda_{1}=0$, so we may drop the common denominator $T_{0}^{2}\left(S_{1}, ., ., S_{K}\right)$ to obtain

$$
0=\left[\sum_{i=1}^{K} G_{i} T_{0}^{2}\left(S_{1}, ., S_{i}=0, ., S_{K}\right)\right] A\left(S_{1}, \ldots, S_{K}\right)-\sum_{i=1}^{K} G_{i} T_{0}^{2}\left(S_{1}, ., S_{i}=0, ., S_{K}\right) A\left(S_{1}, . .,-S_{i}, . ., S_{K}\right)
$$

In the disordered case, we expect that the dynamical transition between the two ferromagnetic states will be dominated by a single dynamical path near zero temperature. So let us now compute the dynamical barrier associated to a given dynamical path.

\section{B. Dynamical barrier associated to a given dynamical path $(1,2, . ., K)$}

In this section, we consider the given dynamical path called $(1,2, . ., K)$

$$
\begin{array}{r}
A\left(-S_{a},-S_{a},-S_{a}, \ldots,-S_{a},-S_{a}\right) \leftrightarrow A\left(S_{a},-S_{a},-S_{a},-S_{a},-S_{a}\right) \leftrightarrow A\left(S_{a}, S_{a},-S_{a}, \ldots,-S_{a},-S_{a}\right) \\
\ldots \leftrightarrow A\left(S_{a}, S_{a}, \ldots, S_{a},-S_{a}\right) \leftrightarrow A\left(S_{a}, S_{a}, S_{a}, S_{a}, S_{a}\right)
\end{array}
$$

Then we are left with a one-dimensional problem with the notations

$$
\begin{aligned}
q(0) & =A\left(-S_{a},-S_{a},-S_{a} \ldots,-S_{a}\right) \simeq-e^{\beta J_{a}} \\
q(1) & =A\left(S_{a},-S_{a},-S_{a}, \ldots,-S_{a}\right) \\
q(2) & =A\left(S_{a}, S_{a},-S_{a}, \ldots,-S_{a}\right) \\
\ldots & \\
q(K-1) & =A\left(S_{a}, S_{a}, \ldots, S_{a},-S_{a}\right) \\
q(K) & =A\left(S_{a}, S_{a}, \ldots S_{a}, S_{a}\right) \simeq e^{-\beta J_{a}}
\end{aligned}
$$


Eq 24 reduces to

$$
\lambda_{1} \simeq G_{K} e^{-\beta J_{K}}\left(1-\frac{q(K-1)}{q(K)}\right)
$$

and Eq. 25] becomes for $1 \leq k \leq K-1$

$$
q(k)=p_{-}(k) q(k-1)+p_{+}(k) q(k+1)
$$

with the probabilities

$$
\begin{aligned}
p_{-}(k) & =\frac{G_{k} T_{0}^{2}\left(S_{a}, \ldots, S_{a}, S_{k}=0,-S_{a},-S_{a}, \ldots,-S_{a}\right)}{G_{k} T_{0}^{2}\left(S_{a}, \ldots, S_{a}, S_{k}=0,-S_{a}, \ldots,-S_{a}\right)+G_{k+1} T_{0}^{2}\left(S_{a}, \ldots, S_{a}, S_{k+1}=0,-S_{a}, \ldots,-S_{a}\right)} \\
p_{+}(k) & =\frac{G_{k+1} T_{0}^{2}\left(S_{a}, \ldots, S_{a}, S_{k+1}=0,-S_{a}, \ldots,-S_{a}\right)}{G_{k} T_{0}^{2}\left(S_{a}, \ldots, S_{a}, S_{k}=0,-S_{a}, \ldots,-S_{a}\right)+G_{k+1} T_{0}^{2}\left(S_{a}, \ldots, S_{a}, S_{k+1}=0,-S_{a}, \ldots,-S_{a}\right)}
\end{aligned}
$$

normalized to $p_{-}(k)+p_{+}(k)=1$.

The solution can be obtained by [8, 26]

$$
q(k)=q(0) \frac{R_{0}(k, K-1)}{R_{0}(0, K-1)}+q(K) \frac{R_{K}(1, k)}{R_{K}(1, K)}
$$

using Kesten variables [27]

$$
\begin{aligned}
R_{0}(K, K-1) & =0 \\
R_{0}(K-1, K-1) & =1 \\
R_{0}(k \leq K-2, K-1) & =1+\sum_{m=k+1}^{K-1} \prod_{n=m}^{K-1} \frac{p_{+}(n)}{p_{-}(n)} \\
R_{0}(0, K-1) & =1+\sum_{m=1}^{K-1} \prod_{n=m}^{K-1} \frac{p_{+}(n)}{p_{-}(n)}=1+\frac{p_{+}(K-1)}{p_{-}(K-1)}+\ldots+\frac{p_{+}(K-1) p_{+}(K-2) \ldots p_{+}(1)}{p_{-}(K-1) p_{-}(K-2) \ldots p_{-}(1)}
\end{aligned}
$$

and

$$
\begin{aligned}
R_{K}(1,0) & =0 \\
R_{K}(1,1) & =1 \\
R_{K}(1, k \geq 2) & =1+\sum_{m=1}^{k-1} \prod_{n=1}^{m} \frac{p_{-}(n)}{p_{+}(n)} \\
R_{K}(1, K) & =1+\sum_{m=1}^{K-1} \prod_{n=1}^{m} \frac{p_{-}(n)}{p_{+}(n)}=1+\frac{p_{-}(1)}{p_{+}(1)}+\frac{p_{-}(1) p_{-}(2)}{p_{+}(1) p_{+}(2)}+\ldots+\frac{p_{-}(1) p_{-}(2) \ldots p_{-}(K-1)}{p_{+}(1) p_{+}(2) \ldots p_{+}(K-1)}
\end{aligned}
$$

Plugging this solution into Eq 28 yields

$$
\begin{aligned}
\lambda_{1} & \simeq G_{K} e^{-\beta J_{K}}\left(1-\frac{R_{K}(1, K-1)}{R_{K}(1, K)}-\frac{q(0)}{q(K)} \frac{1}{R_{0}(0, K-1)}\right) \\
& \simeq G_{K} e^{-\beta J_{K}} \frac{1+e^{2 \beta J_{a}}}{R_{0}(0, K-1)}
\end{aligned}
$$

so the renormalized amplitude of Eq. 18 becomes

$$
G_{R}=\frac{\lambda_{1}}{2 \cosh \beta J_{a}}=G_{K} e^{\beta\left(J_{a}-J_{K}\right)} \frac{1}{R_{0}(0, K-1)}
$$

So using Eq. 19 we need to compute the ratios of the two probabilities of Eq. 30

$$
\frac{p_{+}(k)}{p_{-}(k)}=\frac{G_{k+1} T_{0}^{2}\left(S_{a}, \ldots, S_{a}, S_{k+1}=0,-S_{a}, \ldots,-S_{a}\right)}{G_{k} T_{0}^{2}\left(S_{a}, \ldots, S_{a}, S_{k}=0,-S_{a}, \ldots,-S_{a}\right)}=\frac{G_{k+1} 2 \cosh \beta\left(\sum_{i=1}^{k} J_{i}-\sum_{i=k+2}^{K} J_{i}+J_{a}\right)}{G_{k} 2 \cosh \beta\left(\sum_{i=1}^{k-1} J_{i}-\sum_{i=k+1}^{K} J_{i}+J_{a}\right)}
$$


as well as the products

$$
\prod_{k=m}^{K-1} \frac{p_{+}(k)}{p_{-}(k)}=\frac{G_{K} T_{0}^{2}\left(S_{a}, S_{a}, \ldots, S_{a}+, S_{K}=0\right)}{G_{m} T_{0}^{2}\left(S_{a}, S_{a}, \ldots, S_{a}, S_{m}=0,-S_{a},-S_{a}, \ldots,-S_{a}\right)}=\frac{G_{K} 2 \cosh \beta\left(\sum_{i=1}^{K-1} J_{i}+J_{a}\right)}{G_{m} 2 \cosh \beta\left(\sum_{i=1}^{m-1} J_{i}-\sum_{i=m+1}^{K} J_{i}+J_{a}\right)}(37)
$$

Our conclusion is thus that the dynamical path $(1,2, . ., K)$ has a renormalized amplitude $G_{R}^{(1,2, . ., K)}$ given by

$$
\begin{aligned}
\frac{1}{G_{R}^{(1,2, . ., K)}} & =\frac{e^{\beta\left(J_{K}-J_{a}\right)}}{G_{K}} R_{0}(0, K-1) \\
& =\frac{e^{\beta\left(J_{K}-J_{a}\right)}}{G_{K}}\left[1+\sum_{m=1}^{K-1} \frac{G_{K} 2 \cosh \beta\left(\sum_{i=1}^{K-1} J_{i}+J_{a}\right)}{G_{m} 2 \cosh \beta\left(\sum_{i=1}^{m-1} J_{i}-\sum_{i=m+1}^{K} J_{i}+J_{a}\right)}\right] \\
& \simeq e^{\beta\left(J_{K}-J_{a}\right)} 2 \cosh \beta\left(\sum_{i=1}^{K-1} J_{i}+J_{a}\right) \sum_{m=1}^{K} \frac{1}{G_{m} 2 \cosh \beta\left(\sum_{i=1}^{m-1} J_{i}-\sum_{i=m+1}^{K} J_{i}+J_{a}\right)} \\
& \simeq e^{\beta \sum_{i=1}^{K} J_{i}} \sum_{m=1}^{K} \frac{1}{G_{m} e^{\beta\left|\sum_{i=1}^{m-1} J_{i}-\sum_{i=m+1}^{K} J_{i}+J_{a}\right|}}
\end{aligned}
$$

In terms of the dynamical barriers near zero temperature introduced in Eq. 11

$$
G_{i} \underset{\beta \rightarrow+\infty}{\propto} e^{-\beta \mathcal{B}_{i}}
$$

Eq. 38 yields that the renormalized barrier $\mathcal{B}_{R}^{(1,2, . ., K)}$ associated to the dynamical path $(1,2, . ., K)$ near zero temperature is given by

$$
\begin{aligned}
\mathcal{B}_{R}^{(1,2, ., K)} & \equiv \lim _{\beta \rightarrow+\infty} \frac{\ln \frac{1}{G_{R}^{(1,2, \ldots, K)}}}{\beta}=\max _{1 \leq m \leq K}\left[\mathcal{B}_{m}+\sum_{i=1}^{K} J_{i}-\left|\sum_{i=1}^{m-1} J_{i}-\sum_{i=m+1}^{K} J_{i}+J_{a}\right|\right] \\
& =\max _{1 \leq m \leq K}\left[\mathcal{B}_{m}+J_{m}-J_{a}+2 \min \left(\sum_{i=1}^{m-1} J_{i}+J_{a} ; \sum_{i=m+1}^{K} J_{i}\right)\right]
\end{aligned}
$$

\section{Optimization over the $K$ ! dynamical paths}

We now have to consider the $K$ ! possible dynamical paths : for a given permutation $\pi$ of the $K$ renormalized spins, the dynamical barrier $\mathcal{B}_{R}^{(\pi(1), \pi(2), . ., \pi(K))}$ associated to the path $(\pi(1), \pi(2), . ., \pi(K))$ reads by adapting Eq40

$$
\mathcal{B}_{R}^{(\pi(1), \pi(2), . ., \pi(K))}=\max _{1 \leq m \leq K}\left[\mathcal{B}_{\pi(m)}+J_{\pi(m)}-J_{a}+2 \min \left(\sum_{i=1}^{m-1} J_{\pi(i)}+J_{a} ; \sum_{i=m+1}^{K} J_{\pi(i)}\right)\right]
$$

We now have to choose the dynamical path, i.e. the permutation $\pi$ leading to the smallest barrier. So the final renormalization rule is that the renormalized barrier $\mathcal{B}_{R}$ is given by the minimum of Eq. 41 over the $K$ ! possible permutations

$$
\begin{aligned}
\mathcal{B}_{R} & \equiv \min _{\pi}\left(\mathcal{B}_{R}^{(\pi(1), \pi(2), . ., \pi(K))}\right) \\
& =\min _{\pi}\left(\max _{1 \leq m \leq K}\left[\mathcal{B}_{\pi(m)}+J_{\pi(m)}-J_{a}+2 \min \left(\sum_{i=1}^{m-1} J_{\pi(i)}+J_{a} ; \sum_{i=m+1}^{K} J_{\pi(i)}\right)\right]\right)
\end{aligned}
$$

\section{Limit of the pure case}

In the pure case, all couplings $J_{i}$ have the same value $J_{0}$ and all renormalized boundary spins $S_{i}$ have also the same barrier $\mathcal{B}_{i}=\mathcal{B}$, so that the renormalization rule of Eq. 42 reduces to

$$
\mathcal{B}_{R}=\mathcal{B}+2 J_{0} \max _{1 \leq m \leq K}[\min (m ; K-m)]
$$


For even $K$, the maximum is reached for $m=K / 2$, whereas for odd $K$, the maximum is reached for $m=(K \pm 1) / 2$, so that Eq. 43 yields

$$
\begin{aligned}
\mathcal{B}_{R} & =\mathcal{B}+K J_{0} & & \text { for even } K \\
& =\mathcal{B}+(K-1) J_{0} & & \text { for odd } K
\end{aligned}
$$

This recurrence thus leads to the following linear growth

$$
\begin{aligned}
\mathcal{B}_{n}-\mathcal{B}_{n=0} & =n K J_{0} & & \text { for even } K \\
& =n(K-1) J_{0} & & \text { for odd } K
\end{aligned}
$$

with the number $n$ of generations, in agreement with the more detailed results of [8] containing explicit combinatorial prefactors coming from the degeneracy of the $K$ ! dynamical paths. The linear growth with the number $n$ of generations is in agreement with previous works of physicists [28 30] and of mathematicians [31 34]. It turns out that the slope $(K-1) J_{0}$ for odd $K$ of Eq. 45 coincides with the slope obtained in [29], where a so-called 'disjoint strategy' is optimal, whereas the slope $K J_{0}$ for even $K$ of Eq. 45] differs from the slope $J(K-1)$ obtained in [28, 29], where a so-called 'non-disjoint strategy' is optimal. We refer to Refs [28-32] for more explanations on the differences between disjoint/non-disjoint strategies. Here it is clear that the renormalization procedure making coherent clusters of spins within sub-trees corresponds to the disjoint strategy. Within the disjoint strategy, the renormalization of Eq. 42 for dynamical barriers is close to the recursions written in Refs [28 32], even if there exists a difference : in our approach based on the quantum perturbation theory of section II C the spin $S$ is considered to be at equilibrium with respect to its neighbors, whereas in previous approaches 28 32], the question of the time where the roots flips with respect to the flips of the sub-trees has been taken into account differently (see the notions of 'directed barriers', of 'anchored barriers', etc...). Let us also mention that the case of 'disorder in the degrees of the nodes' has been studied by Henley [28], via the model of critical percolation on the regular Cayley tree.

\section{RG FLOW FOR THE PROBABILITY DISTRIBUTION OF DYNAMICAL BARRIERS}

\section{A. Recurrence for the joint distribution of dynamical barriers and couplings}

The renormalization rule of Eq. 42 for dynamical barriers yields that the joint probability $\operatorname{distribution} \mathcal{P}_{n}(\mathcal{B}, J)$ of the barrier $B$ and the coupling $J$ evolves according to the following iteration in terms of the probability distribution $\rho(J)$ of the random ferromagnetic couplings

$$
\begin{aligned}
\mathcal{P}_{n+1}\left(\mathcal{B}_{R}, J_{a}\right)= & \rho\left(J_{a}\right)\left(\prod_{i=1}^{K} \int d \mathcal{B}_{i} d J_{i} \mathcal{P}_{n}\left(\mathcal{B}_{i}, J_{i}\right)\right) \\
\delta & {\left[\mathcal{B}_{R}+J_{a}-\min _{\pi}\left(\max _{1 \leq m \leq K}\left[\mathcal{B}_{\pi(m)}+J_{\pi(m)}+2 \min \left(\sum_{i=1}^{m-1} J_{\pi(i)}+J_{a} ; \sum_{i=m+1}^{K} J_{\pi(i)}\right)\right]\right)\right] }
\end{aligned}
$$

with the partial normalization

$$
\int d \mathcal{B} P_{n}(\mathcal{B}, J)=\rho(J)
$$

The initial condition for the Glauber dynamics of Eq. [6 near zero temperature $G_{G l a u b e r}^{\text {ini }}[h]=\frac{1}{2 \cosh (\beta h)} \simeq e^{-\beta|h|}$ corresponds for an initial boundary spin $S_{i}$ of the tree submitted to the local field $h=J_{i} S$ to the dynamical barrier $\mathcal{B}_{i}=J_{i}$, so that the joint distribution at generation zero reads

$$
\mathcal{P}_{n=0}^{\text {glauber }}(\mathcal{B}, J)=\rho(J) \delta(\mathcal{B}-J)
$$

The form of Eq. 46 actually shows that it is more convenient to replace the variable $\mathcal{B}$ by $B=\mathcal{B}+J$, so that the joint distribution $P_{n}(B, J)$ evolves according to

$$
\begin{aligned}
P_{n+1}\left(B, J_{a}\right)= & \rho\left(J_{a}\right)\left(\prod_{i=1}^{K} \int d B_{i} d J_{i} P_{n}\left(B_{i}, J_{i}\right)\right) \\
& \delta\left[B-\min _{\pi}\left(\max _{1 \leq m \leq K}\left[B_{\pi(m)}+2 \min \left(\sum_{i=1}^{m-1} J_{\pi(i)}+J_{a} ; \sum_{i=m+1}^{K} J_{\pi(i)}\right)\right]\right)\right]
\end{aligned}
$$


with the same partial normalization as in Eq. 47

$$
\int d B P_{n}(B, J)=\rho(J)
$$

and the initial condition (Eq. 48)

$$
P_{n=0}^{\text {glauber }}(B, J) \quad=\rho(J) \delta(B-2 J)
$$

\section{B. Recurrence for integrated probability distributions}

To factorize the minimum and maximum functions involved in the delta function of Eq. 49, it is convenient to introduce the two complementary integrated distributions

$$
\begin{aligned}
F_{n}(B, J) & \equiv \int_{B}^{+\infty} d B^{\prime} P_{n}\left(B^{\prime}, J\right) \\
G_{n}(B, J) & \equiv \int_{-\infty}^{B} d B^{\prime} P_{n}\left(B^{\prime}, J\right)=\rho(J)-F_{n}(B, J)
\end{aligned}
$$

as well as the notation

$$
S_{\pi(m)}^{(\pi)} \equiv 2 \min \left(\sum_{i=1}^{m-1} J_{\pi(i)}+J_{a} ; \sum_{i=m+1}^{K} J_{\pi(i)}\right)
$$

Then the recurrence of Eq. 49 yields

$$
\begin{aligned}
F_{n+1}\left(B, J_{a}\right) & \equiv \int_{B}^{+\infty} d B^{\prime} P_{n+1}\left(B^{\prime}, J_{a}\right) \\
& =\rho\left(J_{a}\right)\left(\prod_{i=1}^{K} \int d B_{i} d J_{i} P_{n}\left(B_{i}, J_{i}\right)\right) \theta\left[B \leq \min _{\pi}\left(\max _{1 \leq m \leq K}\left[\mathcal{B}_{\pi(m)}+S_{\pi(m)}^{(\pi)}\right]\right)\right] \\
& =\rho\left(J_{a}\right)\left(\prod_{i=1}^{K} \int d B_{i} d J_{i} P_{n}\left(B_{i}, J_{i}\right)\right) \prod_{\pi} \theta\left(B \leq \max _{1 \leq m \leq K}\left[B_{\pi(m)}+S_{\pi(m)}^{(\pi)}\right]\right)
\end{aligned}
$$

Let us consider that we have ordered the $K$ ! permutations $\pi$, so that the last product of Eq. 54 can be expanded as

$$
\begin{aligned}
\prod_{\pi} \theta\left[B \leq \max _{1 \leq m \leq K}\left[B_{\pi(m)}+S_{\pi(m)}^{(\pi)}\right]\right] & =\prod_{\pi}\left(1-\theta\left[B \geq \max _{1 \leq m \leq K}\left[B_{\pi(m)}+S_{\pi(m)}^{(\pi)}\right]\right]\right) \\
& =1+\sum_{p=1}^{K !}(-1)^{p} \sum_{\left\{\pi_{1}<\pi_{2}<. .<\pi_{p}\right\}} \prod_{q=1}^{p} \theta\left[B \geq \max _{1 \leq m \leq K}\left[B_{\pi_{q}(m)}+S_{\pi_{q}(m)}^{\left(\pi_{q}\right)}\right]\right] \\
& =1+\sum_{p=1}^{K !}(-1)^{p} \sum_{\left\{\pi_{1}<\pi_{2} . .<\pi_{p}\right\}} \prod_{q=1}^{p} \prod_{m=1}^{K} \theta\left[B \geq B_{\pi_{q}(m)}+S_{\pi_{q}(m)}^{\left(\pi_{q}\right)}\right]
\end{aligned}
$$

For each permutation $\pi_{q}$, we may replace the product over $m=1,2, \ldots, K$, by the product over $k=\pi_{q}(m)$ to obtain

$$
\begin{aligned}
\prod_{\pi} \theta\left[B \leq \max _{1 \leq m \leq K}\left[B_{\pi(m)}+S_{\pi(m)}^{(\pi)}\right]\right] & =1+\sum_{p=1}^{K !}(-1)^{p} \sum_{\left\{\pi_{1}<\pi_{2} . .<\pi_{p}\right\}} \prod_{q=1}^{p} \prod_{k=1}^{K} \theta\left[B \geq B_{k}+S_{k}^{\left(\pi_{q}\right)}\right] \\
& =1+\sum_{p=1}^{K !}(-1)^{p} \sum_{\left\{\pi_{1}<\pi_{2} . .<\pi_{p}\right\}} \prod_{k=1}^{K} \prod_{q=1}^{p} \theta\left[B \geq B_{k}+S_{k}^{\left(\pi_{q}\right)}\right] \\
& =1+\sum_{p=1}^{K !}(-1)^{p} \sum_{\left\{\pi_{1}<\pi_{2}<. .<\pi_{p}\right\}} \prod_{k=1}^{K} \theta\left[B \geq B_{k}+\max _{1 \leq q \leq p} S_{k}^{\left(\pi_{q}\right)}\right]
\end{aligned}
$$


Then Eq 54 becomes

$$
\begin{aligned}
F_{n+1}\left(B, J_{a}\right) & =\rho\left(J_{a}\right)\left(\prod_{i=1}^{K} \int d B_{i} d J_{i} P_{n}\left(B_{i}, J_{i}\right)\right)\left[1+\sum_{p=1}^{K !}(-1)^{p} \sum_{\left\{\pi_{1}<\pi_{2} . .<\pi_{p}\right\}} \prod_{k=1}^{K} \theta\left[B \geq B_{k}+\max _{1 \leq q \leq p} S_{k}^{\left(\pi_{q}\right)}\right]\right] \\
& =\rho\left(J_{a}\right)+\rho\left(J_{a}\right) \sum_{p=1}^{K !}(-1)^{p} \sum_{\left\{\pi_{1}<\pi_{2}<. .<\pi_{p}\right\}}\left(\prod_{k=1}^{K} \int d B_{k} d J_{k} P_{n}\left(B_{k}, J_{k}\right) \theta\left[B_{k} \leq B-\max _{1 \leq q \leq p} S_{k}^{\left(\pi_{q}\right)}\right]\right) \\
& =\rho\left(J_{a}\right)+\rho\left(J_{a}\right) \sum_{p=1}^{K !}(-1)^{p} \sum_{\left\{\pi_{1}<\pi_{2}<.<\pi_{p}\right\}}\left(\prod_{k=1}^{K} d J_{k} G_{n}\left(B-\max _{1 \leq q \leq p} S_{k}^{\left(\pi_{q}\right)}, J_{k}\right)\right)
\end{aligned}
$$

So we obtain the following closed recurrence for the integrated probability $G_{n}$ of Eq. 52

$$
G_{n+1}\left(B, J_{a}\right)=-\rho\left(J_{a}\right) \sum_{p=1}^{K !}(-1)^{p} \sum_{\left\{\pi_{1}<\pi_{2} .<\pi_{p}\right\}}\left(\prod_{k=1}^{K} \int d J_{k} G_{n}\left(B-\max _{1 \leq q \leq p} S_{k}^{\left(\pi_{q}\right)}, J_{k}\right)\right)
$$

with the initial condition of Eq. 51

$$
\begin{aligned}
G_{n=0}^{\text {glauber }}(B, J)=\int_{-\infty}^{B} d b P_{n=0}^{\text {glauber }}(b, J)=\rho(J) \theta(B-2 J) & =0 \text { for } B<2 J \\
& =\rho(J) \text { for } B>2 J
\end{aligned}
$$

\section{Traveling-wave solution}

For disordered models defined on Cayley trees, it is very common to find that the probability distribution $P_{n}(A)$ of some observable $A$ propagates with a speed $v$ and a fixed shape $P^{*}$ as the number $n$ of generations grows

$$
P_{n}(A) \simeq P^{*}(A-n v)
$$

This means that the average value grows linearly with $n$, whereas the width around this averaged value remains finite. This property was discovered by Derrida and Spohn [35] on the specific example of the directed polymer in a random medium, where the observable $A$ of interest is the free-energy, and was then found in various other statistical physics models defined on Cayley trees [36]. This traveling-wave propagation of probability distributions have also been found in quantum models defined on Cayley trees, in particular in the Anderson localization problem [37 41]. The conclusion is thus that the recursion relations that can be written for observables of disordered models defined on trees naturally lead to the traveling wave propagation of the corresponding probability distributions.

So here, it is natural to expect that the solution of Eq. 58 starting from the initial condition of Eq. 59 will be a traveling-wave with some velocity $v$

$$
G_{n}(B, J) \simeq G^{*}(b \equiv B-n v, J)
$$

where the stable shape $G^{*}(b, J)$ of the front satisfies the equation

$$
G^{*}\left(b-v, J_{a}\right)=-\rho\left(J_{a}\right) \sum_{p=1}^{K !}(-1)^{p} \sum_{\left\{\pi_{1}<\pi_{2} . .<\pi_{p}\right\}}\left(\prod_{k=1}^{K} \int d J_{k} G^{*}\left(b-\max _{1 \leq q \leq p} S_{k}^{\left(\pi_{q}\right)}, J_{k}\right)\right)
$$

and the following boundary conditions at infinity

$$
\begin{aligned}
& G^{*}(b, J) \underset{b \rightarrow-\infty}{\simeq} 0 \\
& G^{*}(b, J) \underset{b \rightarrow+\infty}{\simeq} \rho(J)
\end{aligned}
$$

This means that a barrier $B_{n}$ for a sub-tree of $n$ generations reads

$$
B_{n}=n v+b
$$

where $b$ is a random variable of order $O(1)$. In the following sections, we have checked numerically for $K=2$ and $K=3$ that the statistics of dynamical barriers indeed follow the traveling-wave form of Eq. 61. 


\section{CASE OF BRANCHING NUMBER $K=2$}

\section{A. Renormalization rule for dynamical barriers}

Here there are only $K !=2$ permutations $\pi_{1}=(1,2)$ and $\pi_{2}=(2,1)$, so the barriers associated to these two permutations are $(\mathrm{Eq} 41)$

$$
\begin{aligned}
& \mathcal{B}_{R}^{(1,2)}=\max \left[\mathcal{B}_{1}+J_{1}-J_{a}+2 \min \left(J_{2}, J_{a}\right) ; \mathcal{B}_{2}+J_{2}-J_{a}\right] \\
& \mathcal{B}_{R}^{(2,1)}=\max \left[\mathcal{B}_{2}+J_{2}-J_{a}+2 \min \left(J_{1}, J_{a}\right) ; \mathcal{B}_{1}+J_{1}-J_{a}\right]
\end{aligned}
$$

and the optimization of Eq. 42 reads

$$
\mathcal{B}_{R}+J_{a} \equiv \min \left(\max \left[\mathcal{B}_{1}+J_{1}+2 \min \left(J_{2}, J_{a}\right) ; \mathcal{B}_{2}+J_{2}\right] ; \max \left[\mathcal{B}_{2}+J_{2}+2 \min \left(J_{1}, J_{a}\right) ; \mathcal{B}_{1}+J_{1}\right]\right)
$$

\section{B. Recurrence for the integrated probability}

For $K=2$, we have to consider the two permutations $\pi_{1}=(1,2)$ and $\pi_{2}=(2,1)$, so the variables of Eq. 53 reads

$$
\begin{aligned}
S_{1}^{\left(\pi_{1}\right)} & =2 \min \left(J_{2}, J_{a}\right) \\
S_{2}^{\left(\pi_{1}\right)} & =0 \\
S_{1}^{\left(\pi_{2}\right)} & =0 \\
S_{2}^{\left(\pi_{2}\right)} & =2 \min \left(J_{1}, J_{a}\right)
\end{aligned}
$$

and Eq 58 becomes

$$
\begin{aligned}
G_{n+1}\left(B, J_{a}\right) & =\rho\left(J_{a}\right) \int d J_{1} \int d J_{2} G_{n}\left(B-S_{1}^{\left(\pi_{1}\right)}, J_{1}\right) G_{n}\left(B-S_{2}^{\left(\pi_{1}\right)}, J_{2}\right) \\
& +\rho\left(J_{a}\right) \int d J_{1} \int d J_{2} G_{n}\left(B-S_{1}^{\left(\pi_{2}\right)}, J_{1}\right) G_{n}\left(B-S_{2}^{\left(\pi_{2}\right)}, J_{2}\right) \\
& -\rho\left(J_{a}\right) \int d J_{1} \int d J_{2} G_{n}\left(B-\max \left(S_{1}^{\left(\pi_{1}\right)}, S_{1}^{\left(\pi_{2}\right)}\right), J_{1}\right) G_{n}\left(B-\max \left(S_{2}^{\left(\pi_{1}\right)}, S_{2}^{\left(\pi_{2}\right)}\right), J_{2}\right) \\
& =\rho\left(J_{a}\right) \int d J_{1} \int d J_{2} G_{n}\left(B-2 \min \left(J_{2}, J_{a}\right), J_{1}\right) G_{n}\left(B, J_{2}\right) \\
& +\rho\left(J_{a}\right) \int d J_{1} \int d J_{2} G_{n}\left(B, J_{1}\right) G_{n}\left(B-2 \min \left(J_{1}, J_{a}\right), J_{2}\right) \\
& -\rho\left(J_{a}\right) \int d J_{1} \int d J_{2} G_{n}\left(B-2 \min \left(J_{2}, J_{a}\right), J_{1}\right) G_{n}\left(B-2 \min \left(J_{1}, J_{a}\right), J_{2}\right)
\end{aligned}
$$

i.e. finally, the integrated probability $G_{n}$ satisfies the recurrence

$$
\left.G_{n+1}\left(B, J_{a}\right)=\rho\left(J_{a}\right) \int d J_{1} \int d J_{2} G_{n}\left(B-2 \min \left(J_{2}, J_{a}\right)\right), J_{1}\right)\left[2 G_{n}\left(B, J_{2}\right)-G_{n}\left(B-2 \min \left(J_{1}, J_{a}\right), J_{2}\right)\right]
$$

\section{Traveling-wave Ansatz}

The traveling-wave Ansatz of Eq. 61 yields the following equation for the stable shape $G^{*}(b, J)$ of the front

$$
\left.G^{*}\left(b-v, J_{a}\right)=\rho\left(J_{a}\right) \int d J_{1} \int d J_{2} G^{*}\left(b-2 \min \left(J_{2}, J_{a}\right)\right), J_{1}\right)\left[2 G^{*}\left(b, J_{2}\right)-G^{*}\left(b-2 \min \left(J_{1}, J_{a}\right), J_{2}\right)\right]
$$

with the boundary conditions of Eq. 63 


\section{Weak-disorder expansion}

For the pure case $\rho(J)=\delta\left(J-J_{0}\right)$ discussed in section $\amalg$, the solution is simply

$$
G_{\text {pure }}^{*}(b, J)=\delta\left(J-J_{0}\right) \theta(b)=\rho(J) \theta(b)
$$

with the velocity of Eqs 44 and 45

$$
v_{\text {pure }}=2 J_{0}
$$

Let us now consider the case of weak disorder, where the disorder distribution $\rho(J)$ displays a small width around $J_{0}$. Then we expect that the width of the barrier distribution will also be small with respect to $\left(2 J_{0}\right)$. In the region where $G^{*}\left(b-v, J_{a}\right)$ is small, the appropriate linearization of Eq. 70 consists in replacing $G^{*}\left(b, J_{2}\right)$ by its asymptotic behavior $\rho\left(J_{2}\right)(\mathrm{Eq} 63)$ and by neglecting $G^{*}\left(b-2 \min \left(J_{1}, J_{a}\right), J_{2}\right)$ that would give a quadratic contribution, so that we obtain in this weak-disorder regime

$$
G^{*}\left(b-v, J_{a}\right) \simeq 2 \rho\left(J_{a}\right) \int d J_{1} \int d J_{2} \rho\left(J_{2}\right) G^{*}\left(b-2 \min \left(J_{2}, J_{a}\right), J_{1}\right)
$$

This means that the integral

$$
g^{*}(b) \equiv \int d J G^{*}(b, J)
$$

satisfies the closed linear equation

$$
g^{*}(b-v) \simeq 2 \int d J_{a} \rho\left(J_{a}\right) \int d J_{2} \rho\left(J_{2}\right) g^{*}\left(b-2 \min \left(J_{2}, J_{a}\right)\right)
$$

The exponential shape of coefficient $\mu$

$$
g^{*}(b) \underset{b \rightarrow-\infty}{\simeq} e^{\mu b}
$$

is then a solution of the linearization of Eq. 75 if the velocity $v(\mu)$ satisfies

$$
e^{-\mu v(\mu)}=2 \int d J_{a} \rho\left(J_{a}\right) \int d J_{2} \rho\left(J_{2}\right) e^{-2 \mu \min \left(J_{2}, J_{a}\right)}
$$

The velocity as a function of $\mu$

$$
v(\mu)=-\frac{1}{\mu} \ln \left[2 \int d J_{a} \rho\left(J_{a}\right) \int d J_{2} \rho\left(J_{2}\right) e^{-2 \mu \min \left(J_{2}, J_{a}\right)}\right]
$$

presents the two limiting behaviors

$$
\begin{aligned}
& v(\mu) \underset{\mu \rightarrow 0}{\simeq}-\frac{\ln 2}{\mu} \rightarrow-\infty \\
& v(\mu) \underset{\mu \rightarrow+\infty}{\simeq} 2 J_{\text {min }}+\frac{\ln \mu}{\mu} \rightarrow 2 J_{\text {min }}
\end{aligned}
$$

where $J_{\min }$ is the minimal value of the disorder distribution $\rho(J)$. Between these two limits, there exists a maximum value $v^{*}$ at $\mu^{*}$ such that $v^{\prime}(\mu)=0$, and this is this velocity $v^{*}$ that will be dynamically selected [42, 43]. It can be computed for various disorder distributions $\rho(J)$. Let us now consider two explicit cases.

\section{Exponential distribution for the disorder}

For the exponential distribution

$$
\rho(J)=\theta\left(J-J_{0}\right) \frac{1}{\Delta} e^{-\frac{J-J_{0}}{\Delta}}
$$


Eq 78 reads

$$
v(\mu)=2 J_{0}+\frac{1}{\mu} \ln \frac{1+\mu \Delta}{2}
$$

We are looking for the point $\mu^{*}$ where this function is maximum

$$
0=v^{\prime}\left(\mu_{*}\right)=-\frac{1}{\mu_{*}^{2}} \ln \left(\frac{1+\mu_{*} \Delta}{2}\right)+\frac{\Delta}{\mu_{*}\left(1+\mu_{*} \Delta\right)}
$$

i.e. $\mu_{*}=x / \Delta$ where $x \simeq 3.31107$ is the root of the numerical equation

$$
\ln \left(\frac{1+x}{2}\right)=\frac{x}{1+x}
$$

The corresponding selected velocity reads

$$
\begin{aligned}
v_{*}=v\left(\mu_{*}\right) & =2 J_{0}+\frac{1}{\mu_{*}} \ln \left(\frac{1+\mu_{*} \Delta}{2}\right) \\
& =2 J_{0}+\frac{\Delta}{x} \ln \left(\frac{1+x}{2}\right) \\
& =2 J_{0}+\frac{\Delta}{1+x}
\end{aligned}
$$

In conclusion, we obtain that the velocity grows linearly for weak disorder $\Delta$

$$
v^{*}=2 J_{0}+0.23195 \Delta
$$

2. Box distribution for the disorder

For the box distribution of width $\Delta$

$$
\rho(J)=\frac{1}{\Delta} \theta\left(J_{0} \leq J \leq J_{0}+\Delta\right)
$$

Eq 78 becomes

$$
v(\mu)=2 J_{0}-\frac{1}{\mu} \ln \left[\frac{e^{-2 \mu \Delta}+2 \mu \Delta-1}{\mu^{2} \Delta^{2}}\right]
$$

The corresponding selected velocity $v^{*}=v\left(\mu^{*}\right)$ (where $\left.v^{\prime}\left(\mu^{*}\right)=0\right)$ grows again linearly for weak disorder $\Delta$

$$
v^{*}=2 J_{0}+\Delta v_{*} \simeq 2+0.206826 \Delta
$$

\section{E. Numerical results obtained via the pool method}

If one wishes to study numerically real trees, one is limited to rather small number $n$ of generations, because the number of sites grows exponentially in $n$. From the point of view of convergence towards stable probability distributions via recursion relations, it is thus better to use the so-called 'pool method' that allows to study much larger number of generations. The pool method has been used for disordered models on trees 37, 40, 41] or on hierarchical lattices [44 46]. The idea of the pool method is the following : at each generation, one keeps the same number $M_{\text {pool }}$ of random variables to represent probability distributions. Within our present framework, the probability distribution $P_{n}(B, J)$ at generation $n$ will be represented by a pool of $M_{\text {pool }}=10^{6}$ couples $\left(B_{i}, J_{i}\right)$. To construct a new couple $\left(B_{R}, J_{a}\right)$ of generation $(n+1)$, one draws $K$ couples $\left(B_{i}, J_{i}\right)$ within the pool of generation $n$ and apply the rule of Eq. 66.

The numerical results obtained via the pool method for the box distribution of the disorder of Eq. 86 with $J_{0}=1$ are shown on Fig. 2. On Fig. 22(a), we display the convergence towards the traveling-wave form for the finite disorder width $\Delta=1$. On Fig. 2 (b), we show that the velocity $v(\Delta)$ as a function of the disorder strength has for tangent the weak-disorder expansion of Eq. 88 near $\Delta \rightarrow 0$. 

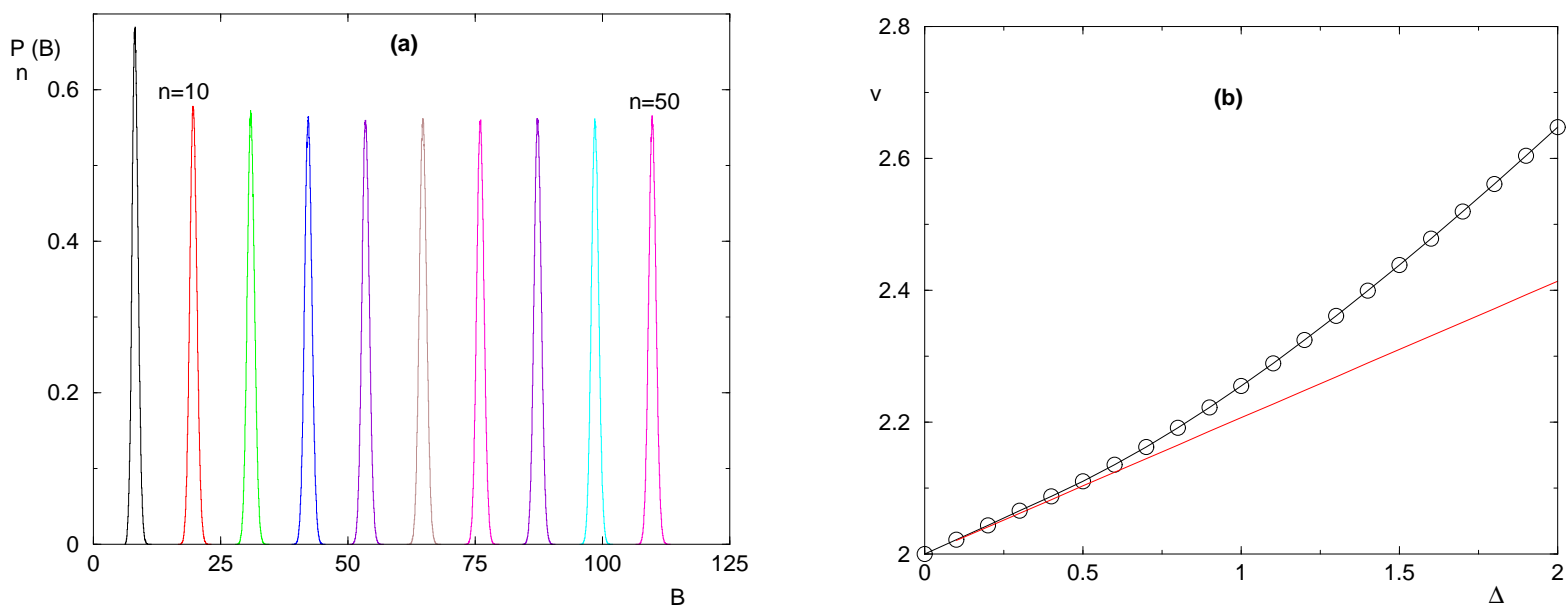

FIG. 2: Cayley tree of branching ratio $K=2$ with the box distribution of disorder of Eq. 86 with $J_{0}=1$ (a) For $\Delta=1$ : the probability distributions $P_{n}(B)$ of the dynamical barrier $B$ at generation $n=5,10,15,20,25,30,35,40,45,50$ correspond to a traveling-wave with a fixed shape $P_{n}(B) \simeq P^{*}(b \equiv B-n v)$ (after an initial transient). (b) Velocity $v$ of the traveling-wave as a function of the disorder width $\Delta$ and agreement with the weak-disorder expansion at first order in $\Delta$ of Eq. 88

\section{CASE OF BRANCHING NUMBER $K=3$}

\section{A. Renormalization rule for dynamical barriers}

Here there are $K !=6$ permutations so the barriers associated to these permutations are (Eq 41)

$$
\begin{array}{ll}
\mathcal{B}_{R}^{\pi_{1}=(1,2,3)} & =\max \left[\mathcal{B}_{1}+J_{1}-J_{a}+2 \min \left(J_{2}+J_{3} ; J_{a}\right) ; \mathcal{B}_{2}+J_{2}-J_{a}+2 \min \left(J_{1}+J_{a}, J_{3}\right) ; \mathcal{B}_{3}+J_{3}-J_{a}\right] \\
\mathcal{B}_{R}^{\pi_{2}=(2,1,3)} & =\max \left[\mathcal{B}_{2}+J_{2}-J_{a}+2 \min \left(J_{1}+J_{3} ; J_{a}\right) ; \mathcal{B}_{1}+J_{1}-J_{a}+2 \min \left(J_{2}+J_{a}, J_{3}\right) ; \mathcal{B}_{3}+J_{3}-J_{a}\right] \\
\mathcal{B}_{R}^{\pi_{3}=(1,3,2)} & =\max \left[\mathcal{B}_{1}+J_{1}-J_{a}+2 \min \left(J_{2}+J_{3} ; J_{a}\right) ; \mathcal{B}_{3}+J_{3}-J_{a}+2 \min \left(J_{1}+J_{a}, J_{2}\right) ; \mathcal{B}_{2}+J_{2}-J_{a}\right] \\
\mathcal{B}_{R}^{\pi_{4}=(3,1,2)} & =\max \left[\mathcal{B}_{3}+J_{3}-J_{a}+2 \min \left(J_{2}+J_{1} ; J_{a}\right) ; \mathcal{B}_{1}+J_{1}-J_{a}+2 \min \left(J_{3}+J_{a}, J_{2}\right) ; \mathcal{B}_{2}+J_{2}-J_{a}\right] \\
\mathcal{B}_{R}^{\pi_{5}=(2,3,1)} & =\max \left[\mathcal{B}_{2}+J_{2}-J_{a}+2 \min \left(J_{1}+J_{3} ; J_{a}\right) ; \mathcal{B}_{3}+J_{3}-J_{a}+2 \min \left(J_{2}+J_{a}, J_{1}\right) ; \mathcal{B}_{1}+J_{1}-J_{a}\right] \\
\mathcal{B}_{R}^{\pi_{6}=(3,2,1)} & =\max \left[\mathcal{B}_{3}+J_{3}-J_{a}+2 \min \left(J_{2}+J_{1} ; J_{a}\right) ; \mathcal{B}_{2}+J_{2}-J_{a}+2 \min \left(J_{3}+J_{a}, J_{1}\right) ; \mathcal{B}_{1}+J_{1}-J_{a}\right]
\end{array}
$$

and the optimization of Eq. 42 reads

$$
\mathcal{B}_{R} \equiv \min \left(\mathcal{B}_{R}^{(1,2,3)} ; \mathcal{B}_{R}^{(2,1,3)} ; \mathcal{B}_{R}^{(1,3,2)}, \mathcal{B}_{R}^{(3,1,2)}, \mathcal{B}_{R}^{(2,3,1)}, \mathcal{B}_{R}^{(3,2,1)}\right)
$$

The corresponding iteration of $\mathrm{Eq} 58$ for the joint probability distribution is rather lengthy and not very illuminating, so we will not write it here, but present instead our numerical results.

\section{B. Numerical results obtained via the pool method}

The numerical results obtained via the pool method for the box distribution of the disorder of Eq. 86 with $J_{0}=1$ are shown on Fig. 3. We find again that the statistics of dynamical barriers is a traveling wave (see Fig. 3 (a)). The corresponding velocity $v(\Delta)$ as a function of the disorder strength $\Delta$ is shown on Fig. 3 (b).

\section{CONCLUSION}

To study the stochastic dynamics near zero-temperature of the random ferromagnetic Ising model on a Cayley tree of branching ratio $K$, we have applied the Boundary Real Space Renormalization procedure introduced in our previous work [8] to derive the renormalization rule for dynamical barriers. The main outcome is that the probability 

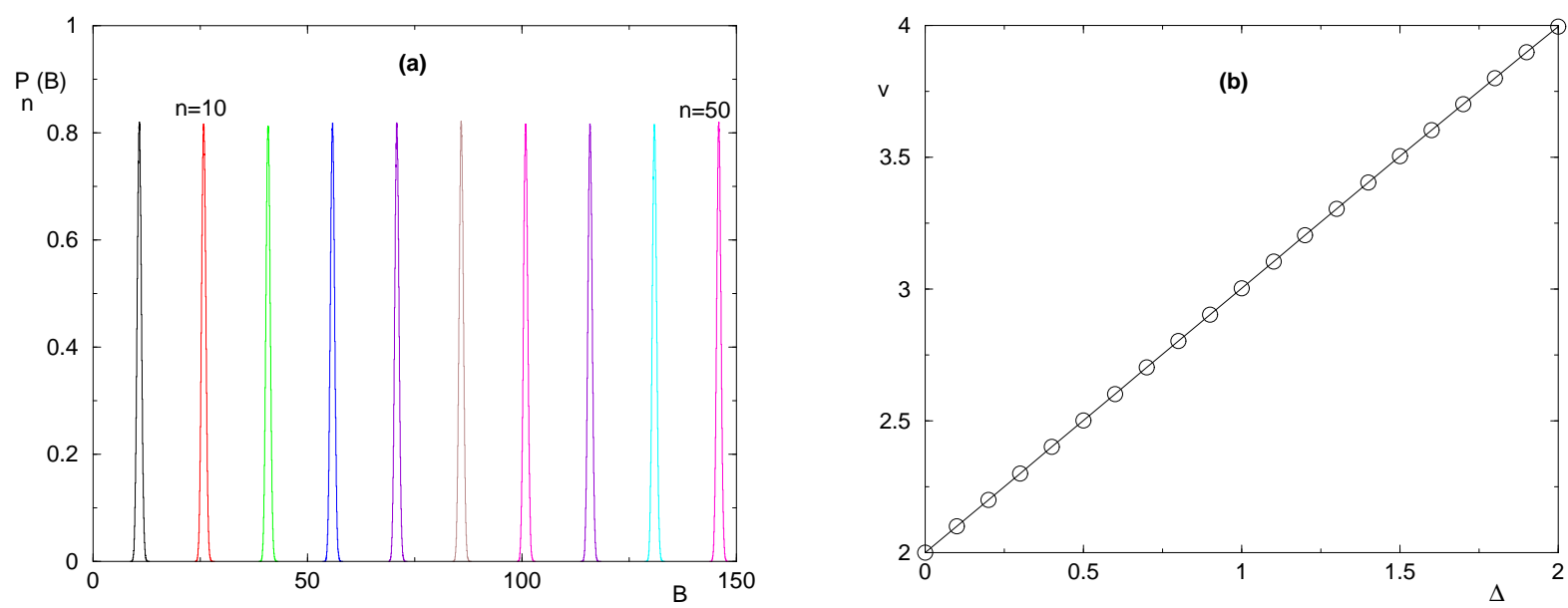

FIG. 3: Cayley tree of branching ratio $K=3$ with the box distribution of disorder of Eq. 86 with $J_{0}=1$ (a) For $\Delta=1:$ the probability distributions $P_{n}(B)$ of the dynamical barrier $B$ at generation $n=5,10,15,20,25,30,35,40,45,50$ correspond to a traveling-wave with a fixed shape $P_{n}(B) \simeq P^{*}(b \equiv B-n v)$. (b) Velocity $v$ of the traveling-wave as a function of the disorder width $\Delta$

distribution $P_{n}(B)$ of dynamical barrier for a subtree of $n$ generations converges for large $n$ towards some travelingwave $P_{n}(B) \simeq P^{*}(B-n v)$, i.e. the width of the probability distribution remains finite around an average-value that grows linearly with the number $n$ of generations. We have presented numerical results for the branching ratios $K=2$ and $K=3$, and we have computed the weak-disorder expansion of the velocity $v$ for $K=2$.

As explained in section IVC the recursion relations that can be written for observables of disordered models defined on trees naturally lead to the traveling wave propagation of probability distributions. So we expect that for other disordered statistical models defined on trees, the statistics of dynamical barriers should be also described by travelling-waves.

[1] "Spin-glasses and random fields", Edited by A.P. Young, World Scientific, Singapore (1998).

[2] "Slow relaxations and non-equilibrium dynamics in Condensed matter", Les Houches July 2002, Edited by J.L. Barrat, M.V. Feigelman, J. Kurchan, J. Dalibard, EDP Les Ulis, Springer, Berlin.

[3] W. Krauth and O. Pluchery, J. Phys. A 27 (1994) L715; W. Krauth, " Introduction to Monte Carlo Algorithms" in 'Advances in Computer Simulation' J. Kertesz and I. Kondor, eds, Lecture Notes in Physics (Springer Verlag, 1998); W. Krauth," Statistical mechanics : algorithms and computations", Oxford University Press (2006).

[4] D. Fisher, P. Le Doussal and C. Monthus, Phys. Rev. Lett. 80 (1998) 3539 ;

D. S. Fisher, P. Le Doussal and C. Monthus, Phys. Rev. E 59 (1999) 4795;

C. Monthus and P. Le Doussal, Physica A 334 (2004) 78.

[5] C. Monthus, Phys. Rev. E 67 (2003) 046109.

[6] C. Monthus and T. Garel, Phys. Rev. E 81, 011138 (2010).

[7] C. Monthus and T. Garel, J. Phys. A Math. Theor. 41, 255002 (2008);

C. Monthus and T. Garel, J. Stat. Mech. P07002 (2008);

C. Monthus and T. Garel, J. Phys. A Math. Theor. 41, 375005 (2008).

[8] C. Monthus and T. Garel, J. Stat. Mech. P02037 (2013).

[9] C. Monthus and T. Garel, J. Stat. Mech. P02023 (2013).

[10] R.J. Glauber, J. Math. Phys. 4, 294 (1963).

[11] B.U. Felderhof, Rev. Math. Phys. 1, 215 (1970); Rev. Math. Phys. 2, 151 (1971).

[12] E. D. Siggia, Phys. Rev. B 16, 2319 (1977).

[13] J. C. Kimball, J. Stat. Phys. 21, 289 (1979).

[14] I. Peschel and V. J. Emery, Z. Phys. B 43, 241 (1981).

[15] C. Monthus and T. Garel, J. Stat. Mech. P12017 (2009).

[16] C. Castelnovo, C. Chamon and D. Sherrington, Phys. Rev. B 81, 184303 (2012).

[17] D. S. Fisher Phys. Rev. Lett. 69, 534 (1992) ; D. S. Fisher Phys. Rev. B 51, 6411 (1995).

[18] D. S. Fisher, Physica A 263, 222 (1999).

[19] O. Motrunich, S.-C. Mau, D. A. Huse, and D. S. Fisher, Phys. Rev. B 61, 1160 (2000).

[20] I. A. Kovacs and F. Igloi, J. Phys. Cond. Matt. 23, 404204 (2011). 
[21] F. Igloi and C. Monthus, Phys. Rep. 412, 277 (2005).

[22] L. B. Ioffe and M. Mezard, Phys. Rev. Lett. 105, 037001 (2010)

[23] M. V. Feigelman, L. B. Ioffe, and M. Mezard, Phys. Rev. B 82, 184534 (2010).

[24] O. Dimitrova and M. Mezard, J. Stat. Mech. (2011) P01020.

[25] C. Monthus and T. Garel, J. Stat. Mech. P09016 (2012).

[26] F. Solomon, Ann. Prob. 3 , 1 (1975); Y.G. Sinai, Theor. Prob. Appl. 27, 256 (1982); B. Derrida and Y. Pomeau, Phys. Rev. Lett. 48, 627 (1982); B. Derrida, J. Stat. Phys. 31, 433 (1983).

[27] H. Kesten, Acta Math. 131, 207 (1973); H. Kesten, M. Koslov, F. Spitzer, Compositio Math 30, 145 (1975); B. Derrida, H.J. Hilhorst, J. Phys. A 16, 2641 (1983); C. Calan, J.M. Luck, T. Nieuwenhuizen, D. Petritis, J. Phys. A 18, 501 (1985).

[28] C.L. Henley, Phys. Rev. B 33, 7675 (1986).

[29] R. Melin, J.C. Angles d'Auriac, P. Chandra and B. Doucot, J. Phys. A Math Gen 29, 5773 (1996); J.C. Angles d'Auriac, M. Preissmann and A. Sebo, Math. Comput. Model. 26, 1 (1997).

[30] A. Montanari and G. Semerjian, J. Stat. Phys. 124, 103 (2006).

[31] T. Lengauer, SIAM J. Alg. Disc. Meth. 3, 99 (1982).

[32] M. Yannakakis, J. A.C.M. 32, 950 (1985).

[33] F. Martinelli, A. Sinclair, D. Weitz, Comm. Math. Phys. 250, 301 (2004).

[34] N. Berger, C. Kenyon, E. Mossel, Y. Peres, Prob. Theory Rel. Fields 131, 311 (2005).

[35] B. Derrida and H. Spohn, J. Stat. Phys. 51, 817 (1988);

J. Cook and B. Derrida, J. Stat. Phys. 63, 505 (1991).

[36] S.N. Majumdar and P.L. Krapivsky, Phys. Rev. E 62, 7735 (2000);

E. Ben-Naim, P L Krapivsky and S N Majumdar, Phys. Rev. E 64035101 (2001);

S.N. Majumdar and P.L. Krapivsky, Phys. Rev. E 65, 036127 (2002)

S.N. Majumdar and P.L. Krapivsky, Physica A 318, 161 (2003);

S.N. Majumdar, Phys. Rev. E 68, 026103 (2003); S N Majumdar, D S Dean and P L Krapivsky, Pramana J. Phys. 64, 1175 (2005).

[37] R. Abou-Chacra, P.W. Anderson and D.J. Thouless, J. Phys. C : Solid State Physics 6, 1734 (1973)

R. Abou-Chacra and D. J. Thouless, J. Phys. C: Solid State Phys. 7, 65 (1974).

[38] M.R. Zirnbauer, Phys. Rev. B 34, 6394 (1986).

[39] K.B. Efetov and O. Viehweger, Phys. Rev. B 45, 11546 (1992);

K.B. Efetov, "Supersymmetry in disorder and chaos", Cambridge University Press (1997).

[40] P.M. Bell and A. MacKinnon, J. Phys. : Condens. Matt. 6, 5423 (1994).

[41] C. Monthus and T. Garel, J. Phys. A: Math. Theor. 42, 075002 (2009).

[42] W. van Saarloos, Phys. Rep. 386, 29 (2003).

[43] E. Brunet, Research lecture on the stochastic Fisher-KPP front equation (Marseille 2007), available at http://www.lps.ens.fr/ ebrunet/

[44] J.R. Banavar and A.J. Bray, Phys. Rev. B 35, 8888 (1987); M. Nifle and H.J. Hilhorst, Phys. Rev. Lett. 68 , 2992 (1992); T. Aspelmeier, A.J. Bray and M.A. Moore, Phys. Rev. Lett. 89, 197202 (2002).

[45] J. Cook and B. Derrida, J. Stat. Phys. 57, 89 (1989).

[46] C. Monthus and T. Garel, Phys. Rev. E 77, 021132 (2008); C. Monthus and T. Garel, Phys. Rev. B 77, 134416 (2008). 